\title{
Dark spectroscopy at lepton colliders
}

\author{
Yonit Hochberg, ${ }^{1,2, *}$ Eric Kuflik, ${ }^{1,2, \dagger}$ and Hitoshi Murayama, ${ }^{3,4,5,+}$ \\ ${ }^{1}$ Department of Physics, LEPP, Cornell University, Ithaca, New York 14853, USA \\ ${ }^{2}$ Racah Institute of Physics, Hebrew University of Jerusalem, Jerusalem 91904, Israel \\ ${ }^{3}$ Ernest Orlando Lawrence Berkeley National Laboratory, University of California, \\ Berkeley, California 94720, USA \\ ${ }^{4}$ Department of Physics, University of California, Berkeley, California 94720, USA \\ ${ }^{5}$ Kavli Institute for the Physics and Mathematics of the Universe (WPI), \\ University of Tokyo Institutes for Advanced Study, University of Tokyo, \\ Kashiwa 277-8583, Japan
}

(Received 28 June 2017; revised manuscript received 5 December 2017; published 22 March 2018)

\begin{abstract}
Rich and complex dark sectors are abundant in particle physics theories. Here, we propose performing spectroscopy of the mass structure of dark sectors via mono-photon searches at lepton colliders. The energy of the mono-photon tracks the invariant mass of the invisible system it recoils against, which enables studying the resonance structure of the dark sector. We demonstrate this idea with several well-motivated models of dark sectors. Such spectroscopy measurements could potentially be performed at Belle II, BES-III and future low-energy lepton colliders.
\end{abstract}

DOI: 10.1103/PhysRevD.97.055030

\section{INTRODUCTION}

The existence of dark matter (DM) is by now well established, though its exact identity is unknown. Theoretical proposals for its particle nature span many orders of magnitude in mass, with various possible mechanisms for setting its relic abundance. Often, DM is part of a larger dark sector, comprised of a wealth of resonances, and can exhibit rich dynamics. Moreover, complex dark sectors can arise in models beyond the Standard Model (SM), irrespective of candidates for dark matter. The possibility to experimentally study the structure of dark sectors is, therefore, an extremely relevant and important task.

In the context of dark sectors, expansive attention has been devoted to dark photons that are kinetically mixed with the SM hypercharge [1]. Constraints from beam dumps, fixed-target experiments, B-factories, stellar environments and colliders have been widely studied in the literature (see e.g. Refs. [2-39], and Ref. [40] for a recent review of this topic). Experiments such as BABAR and those at LEP or the LHC have access to the light states of a dark sector by searching for events with missing energy.

\footnotetext{
*yonit.hochberg@cornell.edu

†kuflik@cornell.edu

†hitoshi@berkeley.edu, hitoshi.murayama@ipmu.jp
}

Published by the American Physical Society under the terms of the Creative Commons Attribution 4.0 International license. Further distribution of this work must maintain attribution to the author(s) and the published article's title, journal citation, and DOI. Funded by SCOAP.
Such searches are generically sensitive to dark sector states, but do not directly probe the spectrum of the dark sector, which can often be rich with dark meson resonances. (For works on measuring different dark sector properties at colliders, see Refs. [41-45].)

Here, we propose a method to probe the resonance spectrum of a dark sector. The idea is simple, and we first draw an analogy from QCD. At an $e^{+} e^{-}$machine, the resonance structure of QCD can be mapped by scanning the center of mass energy of the collision. Similarly, the resonance spectrum can be studied by looking at $e^{+} e^{-} \rightarrow$ $\gamma+$ hadrons events at a fixed center of mass energy collision. There, the mono-photon energy traces the mass of the system it recoils against, thus performing spectroscopy even at fixed center of mass energy. As the observation of the resonances is not required, such a measurement can easily be performed on a dark sector, where the resonances may be invisible. A schematic description of this proposed dark spectroscopy is given in Fig. 1.

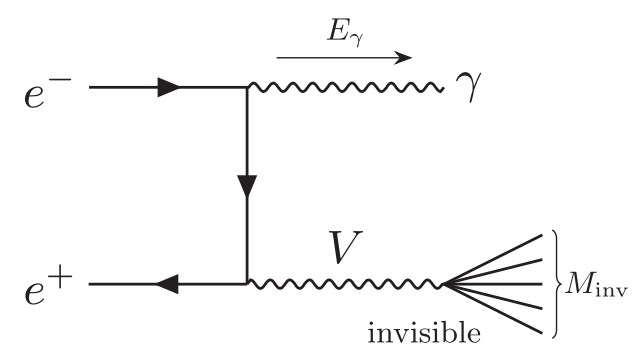

FIG. 1. Mono-photon production at a lepton collider. 
We propose, and study the feasibility of, performing spectroscopy of generic dark sectors at low-energy colliders. The specific case of a strongly interacting massive particle (SIMP) dark sector [46,47], was previously considered by the authors in Ref. [43]. However, the concept outlined in Ref. [43] applies more broadly to any strongly coupled dark sector that interacts with the SM, regardless of the nature of dark matter within the model. It is the purpose of this paper to demonstrate this in a concrete manner.

\section{CONCEPT}

In a $\ell^{+} \ell^{-} \rightarrow \gamma+$ inv collision at center-of-mass energy $\sqrt{s}$, the energy of the outgoing photon is in one-to-one correspondence with the invariant mass of the invisible system, $M_{\mathrm{inv}}$, it recoils against:

$$
E_{\gamma}=\frac{\sqrt{s}}{2}\left(1-\frac{M_{\mathrm{inv}}^{2}}{s}\right)
$$

Thus, by measuring the photon energy, one can determine the spectrum of the undetected system in the process.

For concreteness, we study the case in which a dark sector communicates with the visible sector via a vector, $V$. In later sections, we will take $V$ to be dark $U(1)_{d}$ photon $\gamma_{d}$, which is kinetically mixed with hypercharge. The monophoton production cross section at a lepton collider can then be written as

$$
\begin{aligned}
& \sigma\left(\ell^{+} \ell^{-} \rightarrow \gamma+\mathrm{inv}\right) \\
& =\frac{3 \alpha}{s} \int d \cos \theta \int d M_{\mathrm{inv}}^{2} \frac{M_{\mathrm{inv}}^{2}}{\left(M_{\mathrm{inv}}^{2}-m_{V}^{2}\right)^{2}+m_{V}^{2} \Gamma^{2}} \\
& \times \frac{\Gamma_{V \rightarrow \mathrm{inv}}\left(M_{\mathrm{inv}}\right)}{M_{\mathrm{inv}}} \frac{\Gamma_{V \rightarrow e^{+} e^{-}}\left(M_{\mathrm{inv}}\right)}{M_{\mathrm{inv}}} \frac{8-8 \beta+3 \beta^{2}+\beta^{2} \cos 2 \theta}{\beta \sin ^{2} \theta},
\end{aligned}
$$

where $\beta=1-M_{\text {inv }}^{2} / s$ and the decay widths are to be computed for $m_{V}=M_{\text {inv }}$, reflecting the off-shell nature of the vector in the process.

The irreducible SM background of $\ell^{+} \ell^{-} \rightarrow \gamma \nu \bar{\nu}$ which proceeds via an off-shell $Z$ is easily obtained from Eq. (2) by taking $m_{V}=m_{Z}$ and replacing the invisible width by the $Z$ width into neutrinos. (Note that the contribution from $W$ fusion is negligible.) Additional backgrounds arise from $\ell^{+} \ell^{-} \rightarrow \gamma \gamma$ (peaked at $M_{\mathrm{inv}}^{2}=0$ ), as well as from $\ell^{+} \ell^{-} \rightarrow$ $\gamma \gamma \gamma$ and $\ell^{+} \ell^{-} \rightarrow \gamma \ell^{+} \ell^{-}$when only one photon is observed due to other particles going undetected down the beam-pipe or in a detector crack. Such backgrounds can potentially be mitigated with knowledge over the location of the detector cracks.

We study the potential of low-energy electron collider experiments, such as Belle II and BES-III, to probe the spectroscopy of the dark sector. Belle II is expected to operate at $\sqrt{s}=10 \mathrm{GeV}$ with $50 \mathrm{ab}^{-1}$ of data and
$\mathcal{O}(1-2 \%)$ energy resolution at large $E_{\gamma}$ [48]. BES-III operates at lower center-of-mass-energy $\sqrt{s} \sim 4 \mathrm{GeV}$, with anticipated $10 \mathrm{fb}^{-1}$ and $\sim 2 \%$ energy resolution at high photon energies [49]. To demonstrate the potential reach of these machines, photon energies are smeared using a Gaussian distribution with a given energy resolution. We will take the photon acceptance to be $|\cos \theta|<\cos 12^{\circ}$, motivated by the geometric coverage of Belle II, between 12 to 157 degrees.

\section{RESULTS}

To demonstrate the ability to perform dark spectroscopy at low-energy lepton colliders, we first consider the QCD spectrum, followed by several well-motivated dark sector scenarios. We emphasize that the properties of the dark sector are unknown; the goal of the measurement is to uncover its structure. The dark sectors we study below are just examples that serve to illustrate in a concrete fashion how dark spectroscopy can be achieved.

\section{A. Standard model QCD}

We begin by examining what the resonances of QCD would look like in mono-photon events when ignoring the hadronics in the process, as if they had decayed "invisibly." (Note that the actual presence of hadronic activity in the QCD process means that QCD events are not an irreducible background to the new physics search we propose.) In the $\mathrm{SM}$, the cross section for the lower resonances are dominated by off-shell photons. ${ }^{1}$ The width of the offshell photon can be found from the total hadronic crosssection in $e^{+} e^{-}$annihilations. Using standard notation, the total cross section at center of mass energy $\sqrt{s}$ is

$$
R(s) \equiv \frac{\sigma\left(e^{+} e^{-} \rightarrow \text { hadron }\right)}{\sigma\left(e^{+} e^{-} \rightarrow \mu^{+} \mu^{-}\right)_{0}},
$$

where the subscript 0 refers to the lowest order QED calculation for massless muons,

$$
\sigma\left(e^{+} e^{-} \rightarrow \mu^{+} \mu^{-}\right)_{0}=\frac{4 \pi \alpha^{2}}{3 s}
$$

The data for $R(s)$ is taken from Refs. [50,51]. By cutting the photon propagator in the diagram, $R(s)$ can be written in terms of the off-shell widths at $m_{\gamma^{*}}=\sqrt{s}$,

\footnotetext{
${ }^{1}$ A low-energy description of the vector meson couplings to leptons, called vector-meson dominance, can be given by an effective meson kinetic-mixing with the photons. In this description, the process proceeds via on off-shell photon that mixes with the hadronic resonance. We will use this to describe production of dark resonances, via an off-shell massive vector, below.
} 


$$
R(s)=\frac{\Gamma_{\gamma^{*} \rightarrow \text { hadrons }}(\sqrt{s})}{\Gamma_{\gamma^{*} \rightarrow \mu^{+} \mu^{-}}(\sqrt{s})} .
$$

The off-shell hadronic width at $M_{\text {inv }}$ to be used in Eq. (2) is

$$
\Gamma_{\gamma^{*} \rightarrow \text { had }}\left(M_{\text {inv }}\right)=R\left(M_{\text {inv }}\right) \frac{\alpha M_{\text {inv }}}{3},
$$

and the off-shell electron width,

$$
\Gamma_{\gamma^{*} \rightarrow e^{+} e^{-}}\left(M_{\mathrm{inv}}\right)=\frac{\alpha M_{\mathrm{inv}}}{3} .
$$

The resulting spectrum for $\sqrt{s}=10 \mathrm{GeV}$ at Belle II with $50 \mathrm{ab}^{-1}$ is shown in the left panel of Fig. 2 as a function of the mono-photon energy. We show the QCD result in red, compared to the smeared cross section given a $1 \%$ energy resolution, shown in blue, as well as the number of events in $50 \mathrm{MeV}$ bins, with Poisson variation. The low-mass resonances show up as one, with the dominant contribution coming from the $\phi$. The higher mass $J / \psi$ and $\psi(2 S)$ resonances are clearly visible in the distribution, though the energy resolution of the machine significantly broadens them.

\section{B. Mirror QCD}

Next, we consider an identical mirror copy of the SM (except the mirror photon is massive), where the low-energy resonance structure of the mirror QCD sector is identical to that of QCD. Such a scenario can be motivated by mirror models [52-54] or models of neutral naturalness [55-62].

We assume that there is kinetic mixing between the SM hypercharge and the massive mirror photon, labeled $B_{\mu}$ and $\mathcal{A}_{\mu}$, respectively,

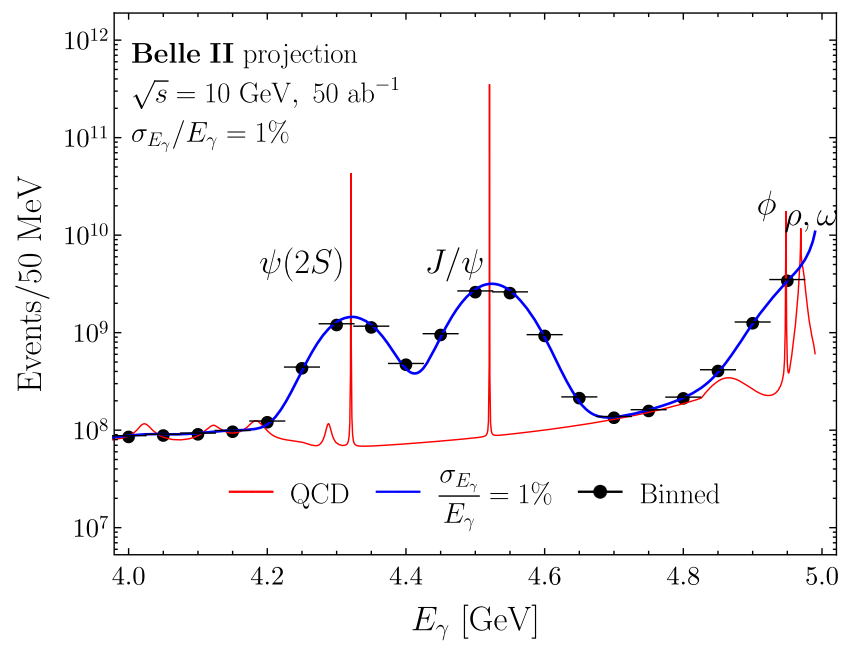

$$
\mathcal{L}=\mathcal{L}_{\mathrm{SM}}+\mathcal{L}_{\text {Mirror }}+\frac{1}{2} m_{\gamma_{d}}^{2} \mathcal{A}_{\mu} \mathcal{A}^{\mu}-\frac{\sin \chi}{2} B_{\mu \nu} \mathcal{A}^{\mu \nu}
$$

Here, the production of mirror mesons proceeds through an additional off-shell mirror photon via the kinetic mixing to the off-shell SM photon. As the mirror sector is identical to the SM-with the same mass spectrum and coupling strengths as in the SM-the rates can be easily obtained. The widths in Eqs. (6) and (7) are modified to the case at hand (see e.g. Ref. [63]),

$$
\begin{aligned}
\Gamma_{\gamma_{d}^{*} \rightarrow \text { inv }}\left(M_{\text {inv }}\right) & =R\left(M_{\text {inv }}\right) \frac{\alpha_{D} M_{\text {inv }}}{3}, \\
\Gamma_{\gamma_{d}^{*} \rightarrow e^{+} e^{-}}\left(M_{\text {inv }}\right) & =\epsilon_{\gamma}^{2} \frac{\alpha M_{\text {inv }}}{3}
\end{aligned}
$$

where $\epsilon_{\gamma} \simeq-c_{w} \sin \chi$. For a full list of conventions, see Sec. 3.1 of Ref. [43].

We take the kinetically mixed mirror photon mass and kinetic mixing parameter to obey all existing constraints (see e.g. Ref. [43]), and show the expected binned distribution at Belle II in right panel of Fig. 2, for $m_{\gamma_{d}}=12 \mathrm{GeV}, \epsilon_{\gamma}=10^{-2}$ and $\alpha_{D}=\alpha$, along with the $\mathrm{SM}$ background. The heavy mirror quarkonium resonances $\psi_{d}(2 S)$ and $J / \psi_{d}$ are clearly visible in the mono-photon distribution, as is the presence of lower resonances around the dark $\phi_{d}$. The narrow states provide a large enhancement in the signal relative to the perturbative prediction if one neglects the resonance structure: for Belle II, the total number of events around the $J / \psi_{d}\left[\psi_{d}(2 S)\right]$ peak is around $\sim 10[\sim 16]$ times larger than the perturbative continuum. A measurement of the cross-section will allow for determination of the mirror quark masses and strong coupling

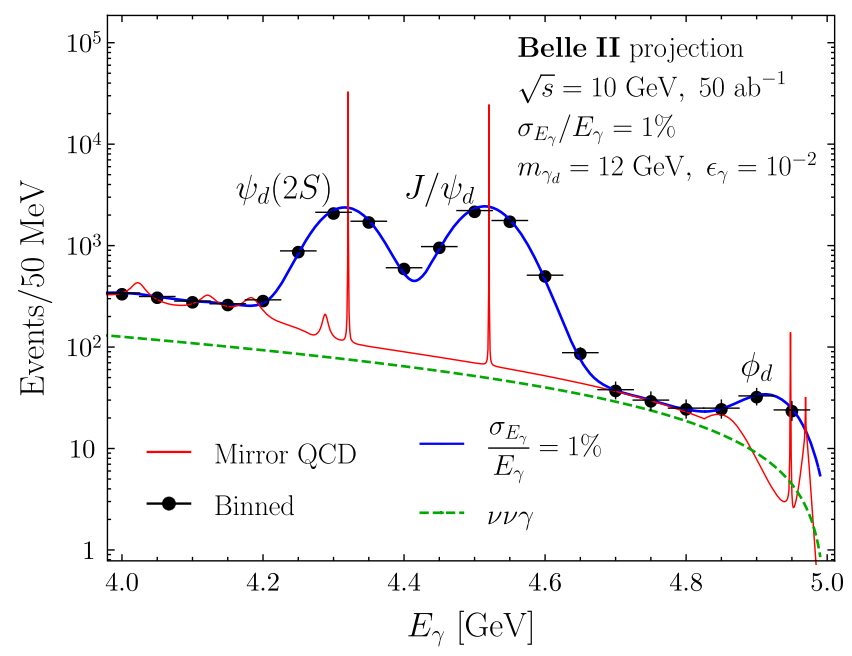

FIG. 2. Left: Distribution for $e^{+} e^{-} \rightarrow \gamma+$ had for QCD, when ignoring the hadronic activity, at $\sqrt{s}=10 \mathrm{GeV}$ at Belle II with 50 ab -1 of data (red), and the expected results for 1\% energy resolution (blue) and binned data (black). Right: The expected distribution for $e^{+} e^{-} \rightarrow \gamma+$ inv for a mirror copy of QCD, with $m_{\gamma_{d}}=12 \mathrm{GeV}$ and $\epsilon_{\gamma}=10^{-2}$, for $50 \mathrm{ab}^{-1}$ at Belle II with $\sqrt{s}=10 \mathrm{GeV}$ (red), $1 \%$ energy resolution (blue), and binned data (black). The SM background of $e^{+} e^{-} \rightarrow \gamma \nu \nu$ is shown in green in both panels. 
constant at the scale of the resonance. We learn that Belle II could shine light on the structure of a mirror QCD.

\section{Dark sectors}

Next, we consider examples of the resonance structure of generic dark sectors such as strongly coupled theories inspired by the models of SIMP dark matter $[46,47]$. In general, we will consider confining gauge theories with a gauged dark $U(1)_{d}$ which kinetically mixes with hyperchage via the Lagrangian Eq. (8). Then production of singlet vector mesons (singlets under both the flavor symmetry and $U(1)_{d}$ ) will proceed via kinetic mixing with the dark photon $\gamma_{d}$. We will refer collectively to the pseudo Nambu-Goldstone bosons as dark pions $\pi_{d}$, and to the light singlet vector mesons that strongly decay as dark rhomesons $\rho_{d}$.

For concreteness of the discussion below, we consider $\operatorname{Sp}\left(N_{c}\right)$ theories with $2 N_{f}$ Weyl fermions in the fundamental representation with degenerate quark masses, so that there are pions on the $S U\left(2 N_{f}\right) / S p\left(2 N_{f}\right)$ coset space and $\rho_{d}$ mesons are in the adjoint of $\operatorname{Sp}\left(2 N_{f}\right)$. This easily generalizes to other gauge theories, with similar phenomenology.

For the $\rho_{d}$ resonance spectrum, we use the partial widths modeled in Ref. [43] as inspired by soft-wall QCD; for simplicity, we summarize the relevant results here. Using the effective meson dominance Lagrangian of $\rho_{d^{-}} \gamma_{d}$ mixing,

$$
\mathcal{L}_{\text {int }}=\frac{F_{\rho_{n}} e_{D}}{2 m_{\rho_{n}}^{2}} \mathcal{A}_{\mu \nu}^{a} \rho_{n}^{\mu \nu a}+\left(e_{D} \mathcal{A}_{\mu}^{a}+g_{\rho} \rho_{\mu}^{a}\right) J^{a \mu},
$$

the spectrum and decay constants are given by

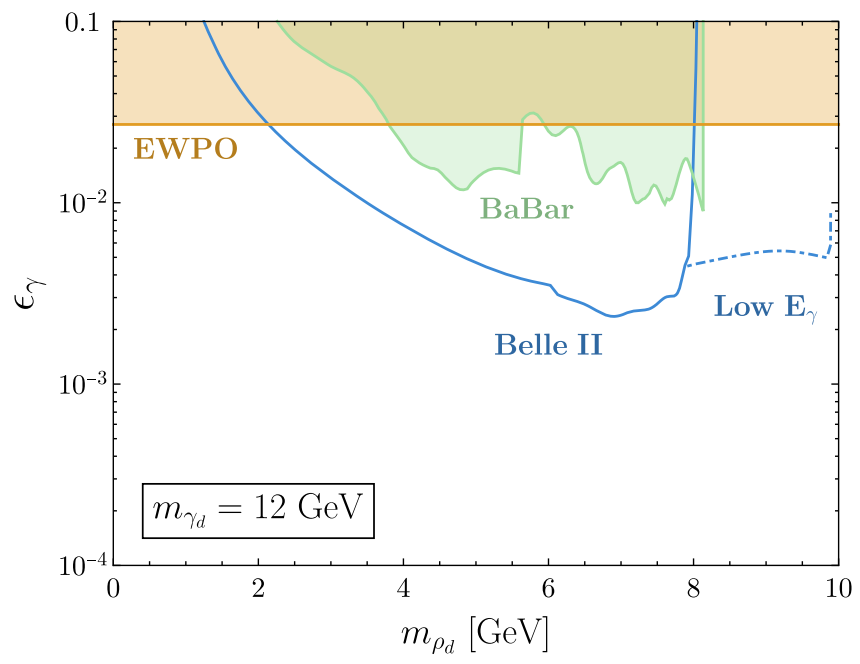

$$
m_{\rho_{n}}^{2}=n m_{\rho_{1}}^{2}, \quad \frac{F_{\rho_{n}}^{2}}{m_{\rho_{n}}^{2}}=\frac{m_{\rho_{1}}^{2}}{2 g_{\rho}^{2}}, \quad g_{\rho}^{2}=\frac{12 \pi^{2}}{N_{c}}
$$

with the $\rho \rightarrow \pi_{d} \pi_{d}$ partial widths of

$$
\Gamma_{n}^{\rho}=\frac{D_{R}}{4} \frac{\beta^{3}}{96 \pi} g_{\rho}^{2} m_{\rho_{n}}, \quad \beta=\sqrt{1-\frac{4 m_{\pi_{d}}^{2}}{m_{\rho_{n}}^{2}}},
$$

assuming all the dark pions $\pi_{d}$ are degenerate. Here, the factor $D_{R}=\operatorname{Tr}\left(T_{\rho}\right)^{2}$ and $T_{\rho}$ is the generator corresponding to the $\rho_{d}$-vector.

Constraints and reach. Next, we estimate the range of parameters in which dark- $\rho$ spectroscopy can be performed. The effective interaction Lagrangian Eq. (10) introduces effective $\rho_{d^{-}} \gamma$ mixing of the size

$\mathcal{L}_{\text {eff }}=\frac{\epsilon_{\rho_{n}}}{2} B_{\mu \nu}^{a} \rho_{n}^{\mu \nu a}, \quad \epsilon_{\rho_{n}} \simeq\left\{\begin{array}{ll}\frac{F_{\rho_{n}} m_{\rho_{n}}^{2} e_{D}}{m_{\gamma_{d}}^{2}} \epsilon_{\gamma} & m_{\gamma_{d}} \gg m_{\rho_{n}} \\ \frac{F_{\rho_{n}} e_{D}}{m_{\rho_{n}}^{2}} \epsilon_{\gamma} & m_{\gamma_{d}} \ll m_{\rho_{n}}\end{array}\right.$.

One can then translate the constraints and estimated reach of a dark photon with mass and kinetic-mixing $\left\{m_{\gamma_{d}}, \epsilon_{\gamma}\right\}$ onto a $\rho_{d}$ meson with mass and kinetic mixing $\left\{m_{\rho_{n}}, \epsilon_{\rho_{n}}\right\}$. These are show in Fig. 3 for high (left) and low (right) $m_{\gamma_{d}}$, for an $S U(2)_{d} \times U(1)_{d}$ gauge theory, with two massless quarks of charge 1 , and $\alpha_{D}=0.1$. We show the constraints from electroweak precision observables (EWPO) [64] and the $B A B A R$ search for dark photons [65], which are independent of the dark sector dynamics and couplings. We also show the translated constraints for $\rho_{d}$ at $B A B A R$ [65], as well as the conservative projections of Ref. [66] for Belle II, which assumes a $1.7 \%$ energy resolution. We note

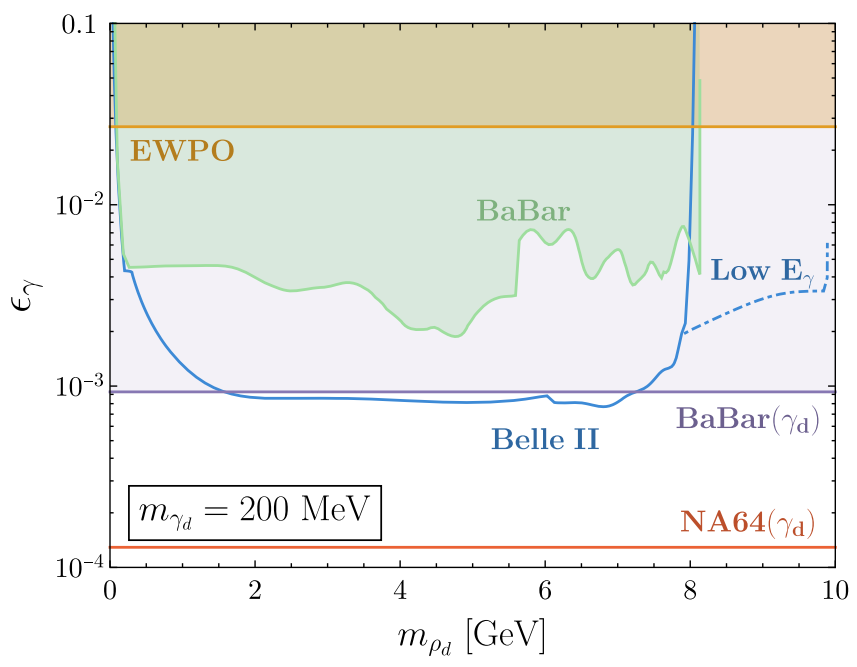

FIG. 3. Constraints on the dark $\rho_{d}$ parameter space, from electroweak precision observables [64] (shaded orange), BABAR search for dark photons [65] (shaded purple), translated BABAR constraints for $\rho_{d}$ [65] (shaded green) and the estimated reach at Belle II [66] (solid and dashed blue curves) and NA64 (solid red) [35], for a heavy (left) and light (right) dark photon. 


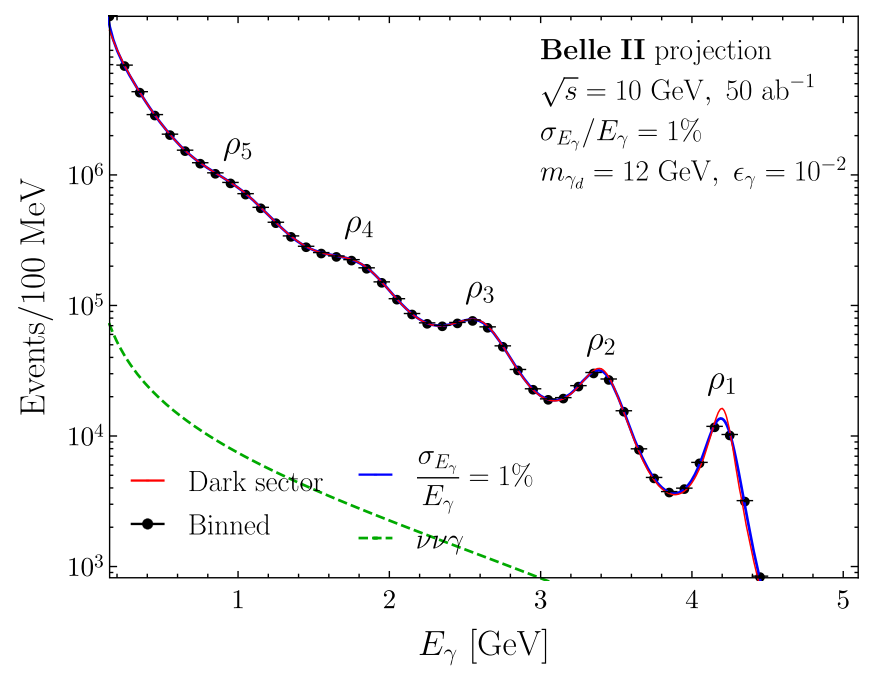

FIG. 4. The cross section for $e^{+} e^{-} \rightarrow \gamma+$ inv at $\sqrt{s}=10 \mathrm{GeV}$ for an $S p(2)_{d} \times U(1)_{d}$ gauge theory with four Weyl fermions, $m_{\gamma_{d}}=12, m_{\pi_{d}}=1, m_{\rho_{1}}=4, \epsilon_{\gamma}=10^{-2}$ and $\alpha_{D}=0.1$, with $1 \%$ energy resolution. The SM background $e^{+} e^{-} \rightarrow \gamma \nu \nu$ is shown in green.

that the expected reach improves with $N_{f}, N_{c}$ and $\alpha_{D}$. We also show the estimated reach of NA64 [35]. Next, in order to resolve the different $\rho_{d}$ peaks from each other, the separation of the peaks need to be larger than the resolution, which imposes $m_{\rho_{1}} \gtrsim \sqrt{s \frac{\sigma_{E_{\gamma}}}{E_{\gamma}}}$. At Belle II, with optimized $1 \%$ energy resolution, this requires $m_{\rho_{d}} \gtrsim 1 \mathrm{GeV}$. Finally, the $\rho_{d}$ bumps should be visible above the continuum dark hadronic production, which is achieved whenever the signal is visible.
Generic dark sector. To exemplify the potential spectroscopy of a generic dark sector, we consider an $S p(2)_{d} \times$ $U(1)_{d}$ gauge theory with $N_{f}=2$ as above [note that $S p(2)$ is $S U(2)]$. In Fig. 4, we show the expected mono-photon energy distribution for $1 \%$ energy resolution, using the expected luminosity of $50 \mathrm{ab}^{-1}$ at Belle II, for the case $m_{\pi_{d}}=1 \mathrm{GeV}, m_{\rho_{1}}=4 \mathrm{GeV}$ and $\epsilon_{\gamma}=10^{-2}$. While the dark photon resonance cannot be seen, the spectrum of resonances is clearly visible at Belle II. This demonstrates that our proposed spectroscopy measurement can be the means through which to discover the dark sector.

SIMPs. Next, we consider a similar symmetry breaking pattern but with a different spectrum. Motivated by SIMP dark matter $[43,46,47]$, where the relic abudance is controlled by $3 \rightarrow 2$ annihilations (and see Refs. [67,68] for contributions of semi-annihilations as well), we take an $S p(4)_{d}$ gauge theory with 4 Weyl fermions, which after confinement gives rise to dark pions $\pi_{d}$ which can play the role of dark matter. We use $m_{\pi_{d}}=500 \mathrm{MeV}, m_{\rho_{1}} / m_{\pi_{d}}=2.1$, $m_{\gamma_{d}}=3 \mathrm{GeV}, \epsilon_{\gamma}=8 \times 10^{-4}$ and $\alpha_{d}=1 /(4 \pi)$, and show the resulting invariant mass distribution for $e^{+} e^{-}$collisions for $\sqrt{s}=10 \mathrm{GeV}$ at Belle II in the left panel of Fig. 5. In this case, the $\rho_{d}$-resonances cannot be resolved at Belle II, but the dark-photon peak is clearly visible. For comparison, we show the distribution for $e^{+} e^{-}$collisions for lower $\sqrt{s}=$ $4 \mathrm{GeV}$ at BES-III (with increased luminosity) in the right panel of Fig. 5, where the resonances are visible. There can also be a kinematic sharp edge at $E_{\gamma}=1.875 \mathrm{GeV}$, corresponding to $M_{\mathrm{inv}}=2 m_{\pi_{d}}$. We learn that low-energy lepton collider experiments such as BES provide complementary tools to higher-energy machines such as Belle II in performing spectroscopy of dark sectors.
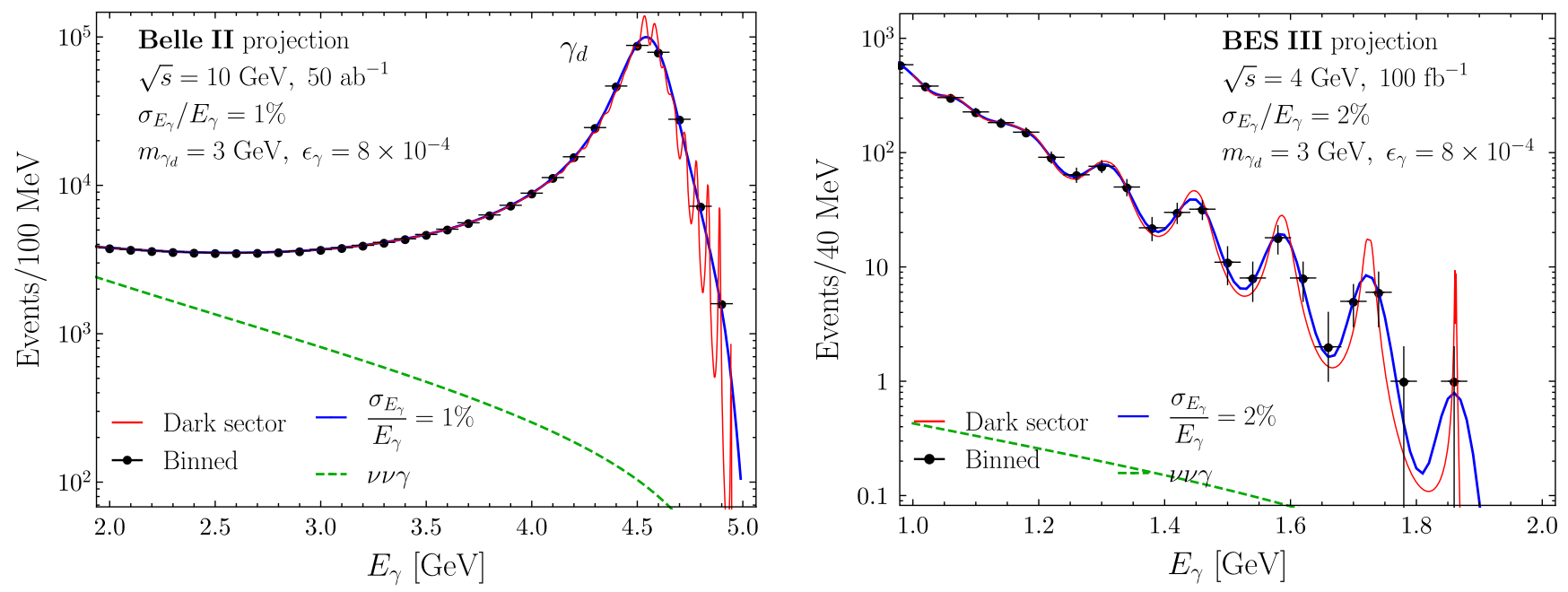

FIG. 5. The cross section for $e^{+} e^{-} \rightarrow \gamma+$ inv for SIMP dark matter of an $S p(4)_{d} \times U(1)_{d}$ gauge theory with 4 Weyl fermions, $m_{\gamma_{d}}=3 \mathrm{GeV}, m_{\pi_{d}}=500 \mathrm{MeV}, m_{\rho_{1}} / m_{\pi_{d}}=2.1, \epsilon_{\gamma}=8 \times 10^{-4}$ and $\alpha_{d}=1 /(4 \pi)$, for Belle II at $\sqrt{s}=10 \mathrm{GeV}$ with $50 \mathrm{ab}^{-1}$ of data (left) and for BES-III with high luminosity of $100 \mathrm{fb}^{-1}$ of data (right), with $1 \%$ and $2 \%$ energy resolution, respectively. The SM background of $e^{+} e^{-} \rightarrow \gamma \nu \nu$ is shown in green in both panels. 


\section{SUMMARY}

In this paper, we have proposed a method to study the spectrum of dark sectors via the measurement of monophoton events at low-energy lepton colliders, such as the Belle II and BES experiments. By considering well-motivated dark sectors, we have shown that such dark spectroscopy can successfully be performed at Belle II, BES and at future lowenergy lepton collider experiments, providing a novel new avenue in which to explore the riches of the dark world.

\section{ACKNOWLEDGMENTS}

We thank Leor Kuflik for inspiration and Maxim Perelstein for comments on the draft. The work of Y. H. is supported by the U.S. National Science Foundation, Grant No. NSF-PHY-1419008, the Large Hadron Collider (LHC) Theory Initiative, the Israel Science Foundation (Grant No. 1112/17) and the Azrieli Foundation. E. K. is supported by the National Science Foundation under Grant No. PHY-1316222, the Israel Science Foundation (Grant No. 1111/17), the Binational Science Foundation (Grant No. 2016153), and the Bethe Postdoctoral Fellowship. H. M. was supported by the U.S. Department of Energy under Contract No. DE-AC02-05CH11231, and by the National Science Foundation under Grants No. PHY-1316783 and No. PHY-1638509. H. M. was also supported by the JSPS Grant-in-Aid for Scientific Research (C) (No. 26400241 and No. 17K05409), MEXT Grant-in-Aid for Scientific Research on Innovative Areas (No. 15H05887 and No. 15K21733), and by World Premier International Research Center Initiative (WPI), Ministry of Education, Culture, Sports, Science and Technology (MEXT), Japan. Y. H. and E. K. were supported by the I-CORE Program of the Planning Budgeting Committee (Grant No. 1937/12). Y. H. and H. M. were supported by Binational Science Foundation (Grant No. 201615).
[1] B. Holdom, Phys. Lett. B 166, 196 (1986).

[2] F. Bergsma et al. (CHARM Collaboration), Phys. Lett. B 166, 473 (1986).

[3] A. Konaka et al., Phys. Rev. Lett. 57, 659 (1986).

[4] E. M. Riordan et al., Phys. Rev. Lett. 59, 755 (1987).

[5] J. D. Bjorken, S. Ecklund, W. R. Nelson, A. Abashian, C. Church, B. Lu, L. W. Mo, T. A. Nunamaker, and P. Rassmann, Phys. Rev. D 38, 3375 (1988).

[6] A. Bross, M. Crisler, S. H. Pordes, J. Volk, S. Errede, and J. Wrbanek, Phys. Rev. Lett. 67, 2942 (1991).

[7] M. Davier and H. Nguyen Ngoc, Phys. Lett. B 229, 150 (1989).

[8] C. Athanassopoulos et al. (LSND Collaboration), Phys. Rev. C 58, 2489 (1998).

[9] P. Astier et al. (NOMAD Collaboration), Phys. Lett. B 506, 27 (2001).

[10] S. Adler et al. (E787 Collaboration), Phys. Rev. D 70, 037102 (2004).

[11] J. D. Bjorken, R. Essig, P. Schuster, and N. Toro, Phys. Rev. D 80, 075018 (2009).

[12] A. V. Artamonov et al. (BNL-E949 Collaboration), Phys. Rev. D 79, 092004 (2009).

[13] R. Essig, R. Harnik, J. Kaplan, and N. Toro, Phys. Rev. D 82, 113008 (2010).

[14] J. Blumlein and J. Brunner, Phys. Lett. B 701, 155 (2011).

[15] S. N. Gninenko, Phys. Lett. B 713, 244 (2012).

[16] J. Blümlein and J. Brunner, Phys. Lett. B 731, 320 (2014).

[17] S. Abrahamyan et al. (APEX Collaboration), Phys. Rev. Lett. 107, 191804 (2011).

[18] H. Merkel et al., Phys. Rev. Lett. 112, 221802 (2014).

[19] H. Merkel et al. (A1 Collaboration), Phys. Rev. Lett. 106, 251802 (2011).

[20] B. Aubert et al. (BABAR Collaboration), Phys. Rev. Lett. 103, 081803 (2009).
[21] D. Curtin et al., Phys. Rev. D 90, 075004 (2014).

[22] J. P. Lees et al. (BABAR Collaboration), Phys. Rev. Lett. 113, 201801 (2014).

[23] G. Bernardi et al., Phys. Lett. B 166, 479 (1986).

[24] R. Meijer Drees et al. (SINDRUM I Collaboration), Phys. Rev. Lett. 68, 3845 (1992).

[25] F. Archilli et al. (KLOE-2 Collaboration), Phys. Lett. B 706, 251 (2012).

[26] S. N. Gninenko, Phys. Rev. D 85, 055027 (2012).

[27] D. Babusci et al. (KLOE-2 Collaboration), Phys. Lett. B 720, 111 (2013).

[28] P. Adlarson et al. (WASA-at-COSY Collaboration), Phys. Lett. B 726, 187 (2013).

[29] G. Agakishiev et al. (HADES Collaboration), Phys. Lett. B 731, 265 (2014).

[30] A. Adare et al. (PHENIX Collaboration), Phys. Rev. C 91, 031901 (2015).

[31] J. R. Batley et al. (NA48/2 Collaboration), Phys. Lett. B 746, 178 (2015).

[32] A. Anastasi et al. (KLOE-2 Collaboration), Phys. Lett. B 757, 356 (2016).

[33] M. Pospelov, Phys. Rev. D 80, 095002 (2009).

[34] J. H. Chang, R. Essig, and S. D. McDermott, J. High Energy Phys. 01 (2017) 107.

[35] S. N. Gninenko, Phys. Rev. D 89, 075008 (2014).

[36] D. Banerjee et al. (NA64 Collaboration), Phys. Rev. Lett. 118, 011802 (2017).

[37] E. Hardy and R. Lasenby, J. High Energy Phys. 02 (2017) 033.

[38] R. Foot and S. Vagnozzi, Phys. Rev. D 91, 023512 (2015).

[39] C. Englert, K. Nordström, and M. Spannowsky, Phys. Rev. D 94, 055028 (2016).

[40] J. Alexander et al., arXiv:1608.08632. 
[41] K. R. Dienes, S. Su, and B. Thomas, Phys. Rev. D 86, 054008 (2012).

[42] K. R. Dienes, S. Su, and B. Thomas, Phys. Rev. D 91, 054002 (2015).

[43] Y. Hochberg, E. Kuflik, and H. Murayama, J. High Energy Phys. 05 (2016) 090.

[44] J. R. Andersen, M. Rauch, and M. Spannowsky, Eur. Phys. J. C 74, 2908 (2014).

[45] H. An, B. Echenard, M. Pospelov, and Y. Zhang, Phys. Rev. Lett. 116, 151801 (2016).

[46] Y. Hochberg, E. Kuflik, T. Volansky, and J. G. Wacker, Phys. Rev. Lett. 113, 171301 (2014).

[47] Y. Hochberg, E. Kuflik, H. Murayama, T. Volansky, and J. G. Wacker, Phys. Rev. Lett. 115, 021301 (2015).

[48] C. Hearty, T. Higuchi, Y. Iwasaki, T. Iwashita, C. Li, and K. Miyabayashi (private communication).

[49] R. A. Briere, F. A. Harris, and R. E. Mitchell, Annu. Rev. Nucl. Part. Sci. 66, 143 (2016).

[50] V. V. Ezhela, S. B. Lugovsky, and O. V. Zenin, arXiv: hep-ph/0312114.

[51] K. A. Olive et al. (Particle Data Group), Chin. Phys. C 38, 090001 (2014).

[52] R. Foot, H. Lew, and R. R. Volkas, Phys. Lett. B 272, 67 (1991).

[53] R. Foot, H. Lew, and R. R. Volkas, Mod. Phys. Lett. A 07, 2567 (1992).

[54] Z. G. Berezhiani, A. D. Dolgov, and R. N. Mohapatra, Phys. Lett. B 375, 26 (1996).
[55] Z. Chacko, H.-S. Goh, and R. Harnik, Phys. Rev. Lett. 96, 231802 (2006).

[56] G. Burdman, Z. Chacko, H.-S. Goh, and R. Harnik, J. High Energy Phys. 02 (2007) 009.

[57] H. Cai, H.-C. Cheng, and J. Terning, J. High Energy Phys. 05 (2009) 045.

[58] N. Craig, S. Knapen, and P. Longhi, Phys. Rev. Lett. 114, 061803 (2015).

[59] N. Craig, S. Knapen, and P. Longhi, J. High Energy Phys. 03 (2015) 106.

[60] Z. Chacko, Y. Nomura, M. Papucci, and G. Perez, J. High Energy Phys. 01 (2006) 126.

[61] B. Batell and M. McCullough, Phys. Rev. D 92, 073018 (2015).

[62] N. Arkani-Hamed, T. Cohen, R. T. D’Agnolo, A. Hook, H. D. Kim, and D. Pinner, Phys. Rev. Lett. 117, 251801 (2016).

[63] B. Batell, M. Pospelov, and A. Ritz, Phys. Rev. D 79, 115008 (2009).

[64] A. Hook, E. Izaguirre, and J. G. Wacker, Adv. High Energy Phys. 2011, 1 (2011).

[65] J. P. Lees et al. (BABAR Collaboration), Phys. Rev. Lett. 119, 131804 (2017).

[66] R. Essig, J. Mardon, M. Papucci, T. Volansky, and Y.-M. Zhong, J. High Energy Phys. 11 (2013) 167.

[67] A. Berlin, N. Blinov, P. Schuster, and N. Toro (to be published).

[68] A. Berlin, P. Schuster, N. Toro, and S. Gori (to be published). 